\title{
Inversion and Zero Dynamics in Nonlinear Multivariable Control
}

\author{
Prodromos Daoutidis and Costas Kravaris \\ Dept. of Chemical Engineering, University of Michigan, Ann Arbor, MI 48109
}

\begin{abstract}
This work concerns general multiple-input/multiple-output (MIMO) nonlinear systems with nonsingular characteristic matrix. For these systems, the problem of inversion is revisited and explicit formulas are derived for the full-order and the reduced inverse system. The reduced inverse naturally leads to an explicit calculation of the unforced zero dynamics of the system and the definition of a concept of forced zero dynamics. These concepts generalize the notion of transmission zeros for MIMO linear systems in a nonlinear setting. Chemical engineering examples are given to illustrate the calculation of zero dynamics. Input/output linearization is then interpreted as canceling the forced zero dynamics of the system, and precise internal stability conditions are derived for the closed-loop system.
\end{abstract}

\section{Introduction}

Nonlinear control has emerged as a research area of rapidly increasing activity during recent years, as a consequence of the realization that the control of severely nonlinear processes must be based on a nonlinear synthesis framework. The development of such a framework requires appropriate mathematical, conceptual, and methodological tools that are able to deal directly with the nonlinear nature of the problems. Differential geometry has provided such tools, indicating a promising direction toward the development of a complete nonlinear control framework. Research in this area started by investigating fundamentai mathematical concepts and has reached a point where basic controller synthesis problems can systematically be solved for a broad class of systems. Input/output linearization for single-input/single-output (SISO) (Kravaris and Chung, 1987) and multiple-input/multiple-output (MIMO) nonlinear systems (Kravaris and Soroush, 1990) has provided an extremely convenient synthesis framework to this end, allowing a unified solution of the disturbance rejection and set-point tracking problem (Daoutidis and Kravaris, 1989, Daoutidis et al., 1990).

From an analysis point of view, internal stability is a key issue for the practical application of the above methods. Intuitively speaking, input/output linearizing feedback generates implicitly an inverse of the system. Consequently, in order for the closed-loop system to be internally stable, the inverse dynamics must be stable in some sense. In a linear setting, stable inverse dynamics is equivalent to the zeros of the system being in the left half-plane, a condition that can be checked easily.
In a nonlinear setting, however, one must check the stability of the inverse itself. This issue has been completely resolved in a SISO nonlinear setting. Hirschorn (1979a) derived an explicit expression for a full-order realization of the inverse dynamics. This realization cannot be used for a stability characterization of the inverse, because it has unstable hidden modes. It allows, however, the calculation of an explicit minimal-order realization of the inverse, which was introduced by Byrnes and Isidori (1985) as the concept of zero dynamics, the nonlinear analog of linear zeros. Kravaris (1988) provided a rigorous interpretation of input/output linearizing feedback as canceling the zero dynamics of the system; this led to precise internal stability conditions for the closed-loop system, expressed through appropriate stability conditions on the zero dynamics.

Intuitively, one would expect that similar results should hold in a MIMO nonlinear setting as well. The derivation of precise internal stability conditions, however, must rely on explicit formulas for the inverse and explicit generalizations of the notion of MIMO zeros in a nonlinear setting. In pursuing this end, important technical difficulties arise. In particular, although nonlinear inversion and zero dynamics have been generalized in a MIMO context in the systems literature (Hirschorn, 1979b; Isidori and Moog, 1988), the construction of the inverse and zero dynamics involves complicated algorithmic procedures and no explicit formulas are available for the general case. 
Based on the above considerations, this work focuses on the class of MIMO nonlinear systems with nonsingular characteristic matrix. The purpose is twofold:

1. To develop explicit formulas for the inverse and zero dynamics

2. To derive precise internal stability conditions for the closed-loop system under input/output linearizing feedback The point of departure for our development is nonlinear inversion. After a brief review of fundamental concepts such as relative order and characteristic matrix, we focus on systems with nonsingular characteristic matrix, for which we derive a formula for a full-order realization of the inverse. A minimalorder realization of the inverse is then easily obtained, working in a convenient normal form representation of the system. This leads to a concept of forced zero dynamics that generalizes the Isidori and Moog concept of (unforced) zero dynamics, and allows a complete stability characterization for the inverse dynamics. Chemical engineering examples are given to illustrate the calculation of the minimal-order inverse and the zero dynamics. Input/output linearization is then interpreted as canceling the forced zero dynamics of a system. This leads naturally to precise internal stability conditions for the closedloop system.

\section{Preliminary Definitions}

We consider general MIMO nonlinear systems with an equal number of inputs and outputs and state-space representation of the form

$$
\begin{aligned}
& \dot{x}=f(x)+\sum_{j=1}^{m} g_{j}(x) u_{j} \\
& y_{i}=h_{i}(x), i=1, \cdots, m
\end{aligned}
$$

where $f$ and $g_{j}$ are smooth vector fields on $\mathbb{R}^{n}, h_{i}$ are smooth scalar fields on $\mathbb{R}^{n}$, and

$$
\boldsymbol{x}=\left[\begin{array}{c}
x_{1} \\
x_{2} \\
\vdots \\
x_{n}
\end{array}\right] \in \mathbb{R}^{n}, \boldsymbol{u}=\left[\begin{array}{c}
u_{1} \\
u_{2} \\
\vdots \\
u_{m}
\end{array}\right] \in \mathbb{R}^{m}, \boldsymbol{y}=\left[\begin{array}{c}
y_{1} \\
y_{2} \\
\vdots \\
y_{m}
\end{array}\right] \in \mathbb{R}^{m}
$$

are the vectors of the states, the manipulated inputs, and the outputs (to be controlled), respectively, with $m \leq n$. In a more compact vector notation, Eq. 1 can take the form:

$$
\begin{aligned}
\dot{x} & =f(x)+g(x) u \\
y_{i} & =h_{i}(x), i=1, \cdots, m
\end{aligned}
$$

where $g(x)$ is a $(n \times m)$ matrix with columns the vector fields $g_{1}(x), \cdots, g_{m}(x)$. For the theoretical development, we assume that the origin is the reference equilibrium point of Eq. 1.

Definition 1. For the MIMO nonlinear system described by Eq. $1, r_{i}$, the relative order of the output $y_{i}$ with respect to the manipulated input vector $u$, is the smallest integer for which

$$
\begin{aligned}
L_{g} L_{f}^{r_{i}-1} h_{i}(x)=\left[L_{g_{1}} L_{f}^{r_{i}-1} h_{i}(x) L_{g_{2}} L_{f}^{r_{i}-1} h_{i}(x)\right. \\
\\
\left.\cdots L_{g_{m}} L_{f}^{r_{i}-1} h_{i}(x)\right] \equiv\left[\begin{array}{llll}
0 & 0 & \cdots & 0
\end{array}\right]
\end{aligned}
$$

In any well-formulated control problem, a finite elative order $r_{i}$ must exist for every $i$. This will guarantee that the control problem is well-posed, in the sense that there 11 be at least one manipulated input variable that affects the output $y_{i}$ through a chain of $r_{i}$ integrations.

Remark 1 . The definition of relative order and consequently any property associated with it is valid, in general, locally in a region around an equilibrium point in state space.

Definition 2. For the MIMO nonlinear system described by Eq. 1, with finite relative orders $r_{1}, \cdots, r_{m}$, the matrix

$$
C(x)=\left[\begin{array}{ccc}
L_{g_{1}} L_{f}^{r_{1}-1} h_{1}(x) & \cdots & L_{k_{m}} L_{f}^{r_{1}-1} h_{1}(x) \\
\vdots & & \vdots \\
L_{g_{1}} L_{f}^{r_{m}-1} h_{m}(x) & \cdots & L_{g_{n}} L_{f}^{r_{m}-1} h_{m}(x)
\end{array}\right]
$$

is called the characteristic matrix of the system.

For the rest of this paper, we will be dealing with MIMO nonlinear systems with nonsingular characteristic matrix. Nonsingularity of the characteristic matrix is a sufficient condition for input/output linearization to be achievable through static state feedback (Kravaris and Soroush, 1990) and, in general, allows a straightforward extension of SISO results in a MIMO context. For systems for which this condition is not satisfied, dynamic state feedback can be employed to transform them into systems for which it is satisfied.

\section{Inversion of Nonlinear Multivariable Systems}

A dynamic system-in general, nonlinear-is said to be invertible at an initial state $x_{0}$, whenever distinct inputs, $u^{1} \neq u^{2}$, result in distinct output values, $y\left(t ; u^{1}, x_{0}\right) \neq y\left(t ; u^{2}, x_{0}\right)$. Thus, given the output-and, in general, the initial state-for an invertible system, one can in principle determine the input applied. A left inverse for the original system would then be a dynamic system which, driven by the process output (and its derivatives), reconstructs the applied input. A right inverse would be a dynamic system which, driven by a desired output trajectory, produces the input necessary to obtain this trajectory. In the case of a square system, one with an equal number of inputs and outputs, the notions of right and left inverse become identical. A realization of the inverse operator can then be interpreted as a right or a left inverse, depending on the context.

The problem of calculating a realization of the inverse has a straightforward solution in the case of linear systems. Given a transfer function description of a linear system, one can easily calculate a realization for the inverse of its transfer function (SISO case) or transfer matrix (MIMO case). In a nonlinear setting however, there is no explicit input/output representation of a system (only in the sense of an abstract operator). Consequently, the calculation of a realization of the inverse becomes a highly nontrivial problem, which necessarily has to be addressed in a state-space framework. Hirschorn (1979a) provided an explicit formula for the inverse of a SISO nonlinear system. The purpose of this section is to derive explicit formulas for a realization of the inverse of a MIMO nonlinear system with a nonsingular characteristic matrix. 


\section{Hirschorn inversion: the full-order inverse}

In the case of a general MIMO system described by Eq. 1, the issue of invertibility is extremely involved. Hirschorn (1979b) suggested an algorithm for the construction of a left inverse that recursively generates a sequence of operators $S_{1}$, $S_{2}, \cdots, S_{k}$, by differentiating the output map. The sequence terminates when the output map for $S_{k}$ can be solved for the manipulated input vector in terms of derivatives of the output. Under certain conditions, invertibility of this map implies invertibility of the original system. In Theorem 1, we show that, for systems with nonsingular characteristic matrix, Hirschorn's inversion algorithm allows the derivation of an explicit formula for the system's inverse; the proof is given in the Appendix.

Theorem 1. Consider a MIMO nonlinear system of the form of Eq. 1, with finite relative orders $r_{i}, i=1, \cdots, m$, and nonsingular characteristic matrix $C(x)$. Then, the dynamic system:

$\dot{\xi}=f(\xi)+g(\xi) C(\xi)^{-1}\left(\left[\begin{array}{c}\frac{d^{r_{1}} y_{1}}{d t^{r_{1}}} \\ \vdots \\ \frac{d^{r_{m}} y_{m}}{d t^{r_{m}}}\end{array}\right]-\left[\begin{array}{c}L_{f}^{r_{1}} h_{1}(\xi) \\ \vdots \\ L_{f}^{r_{m}} h_{m}(\xi)\end{array}\right]\right)$

$u=C(\xi)^{-1}\left(\left[\begin{array}{c}\frac{d^{r_{1}} y_{1}}{d t^{r_{1}}} \\ \vdots \\ d^{r_{m}} y_{m} \\ d t^{r_{m}}\end{array}\right]-\left[\begin{array}{c}L_{f}^{r_{1}} h_{1}(\xi) \\ \vdots \\ L_{f}^{r_{m}} h_{m}(\xi)\end{array}\right]\right)$

is a realization of the inverse of the original system.

Remark 2. In the case of a SISO nonlinear system $(m=1)$ with relative order $r$, the inverse given by Eq. 5 reduces to:

$$
\begin{aligned}
& \dot{\xi}=f(\xi)+g(\xi) \frac{\frac{d^{r} y}{d t^{r}}-L_{f}^{r} h(\xi)}{L_{g} L_{f}^{r-1} h(\xi)} \\
& u=\frac{\frac{d^{r} y}{d t^{r}}-L_{f}^{r} h(\xi)}{L_{g} L_{f}^{r-1} h(\xi)}
\end{aligned}
$$

which, as expected, is exactly the formula for the inverse of a SISO nonlinear system originally derived by Hirschorn (1979a).

An important observation for the realization of the inverse system given by Eq. 5 is that its dynamics is described by $n$ differential equations, and therefore the realization is of full order. A closer inspection of Eq. 5 also reveals that the above Hirschorn inverse involves, by construction, pole-zero cancellations at the origin, with obvious consequences on its internal stability. For the purpose of studying the stability characteristics of the inverse, however, and in analogy with SISO nonlinear systems, one would like to obtain a minimalorder realization of the inverse dynamics.

\section{Normal form and the reduced inverse}

Isidori and Moog (1988) provided an algorithm for the calculation of a reduced inverse, that is, a minimal-order realization of the inverse, in the general case of a MIMO nonlinear system. It is our purpose to derive an explicit formula for such a realization in the case of a system with nonsingular characteristic matrix. The result of Theorem 1 can be used to obtain such a formula, provided that the system of Eq. 1 is transformed in an appropriate coordinate system. For this reason, we will now review the so-called normal form representation of a MIMO nonlinear system.

Proposition 1 (Isidori, 1989). For a nonlinear system described by Eq. 1, with finite relative orders $r_{i}, i=1, \cdots, m$ and nonsingular characteristic matrix $C(x)$, the scalar fields

$$
\begin{aligned}
h_{1}(x), L_{f} h_{1}(x), \cdots, L_{f}^{r_{1}-1} h_{1}(x), \cdots & \\
& h_{m}(x), L_{f} h_{m}(x), \cdots, L_{f}^{r_{m}-1} h_{m}(x)
\end{aligned}
$$

are linearly independent.

An immediate consequence of the above proposition is that

- $r_{1}+r_{2}+\cdots+r_{m} \leq n$

- the scalar fields

$$
\begin{aligned}
h_{1}(x), L_{f} h_{1}(x), \cdots, L_{f}^{r_{1}-1} h_{1}(x), \cdots, & \\
& h_{m}(x), L_{f} h_{m}(x), \cdots, L_{f}^{r_{m}-1} h_{m}(x)
\end{aligned}
$$

qualify as a part of a curvilinear coordinate system.

Furthermore, one can always find scalar fields $t_{1}(x), \cdots$, $t_{n-\Sigma_{i r},}(x)$ such that the scalar fields

$$
\begin{aligned}
t_{1}(x), \cdots, t_{n-\Sigma_{i} r_{r}}(x), h_{1}(x), L_{f} h_{1}(x), \cdots, L_{f}^{r_{1}-1} h_{1}(x), \cdots, \\
h_{m}(x), L_{f} h_{m}(x), \cdots, L_{f}^{r_{m}-1} h_{m}(x)
\end{aligned}
$$

are linearly independent. Then the transformation:

$$
\zeta=\left[\begin{array}{c}
\zeta^{(0)} \\
-- \\
\zeta^{(1)} \\
-- \\
\vdots \\
-- \\
\zeta^{(m)}
\end{array}\right]=\Phi(x)=\left[\begin{array}{c}
t_{1}(x) \\
\vdots \\
t_{n-\Sigma_{i} r_{i}}(x) \\
\cdots h_{1}(x) \\
L_{f} h_{1}(x) \\
\vdots \\
L_{f}^{r_{1}-1} h_{1}(x) \\
-\cdots---- \\
\vdots \\
-h_{m}(x) \\
L_{f} h_{m}(x) \\
\vdots \\
L_{f}^{r_{m}-h_{m}(x)}
\end{array}\right]
$$

is invertible and qualifies as a curvilinear coordinate system. The original system transformed in the new coordinates takes the form:

$$
\begin{aligned}
\dot{\zeta}_{1}^{(0)} & =F_{1}\left(\zeta^{(0)}, \zeta^{(1)}, \cdots, \zeta^{(m)}\right)+G_{1}\left(\zeta^{(0)}, \zeta^{(1)}, \cdots, \zeta^{(m)}\right) u \\
& \vdots \\
\dot{\zeta}_{n-\Sigma_{i} r_{1}}^{(0)} & =F_{n-\Sigma_{i} r_{i}}\left(\zeta^{(0)}, \zeta^{(1)}, \cdots, \zeta^{(m)}\right) \\
& \quad+G_{n-\Sigma_{i} r_{i}}\left(\zeta^{(0)}, \zeta^{(1)}, \cdots, \zeta^{(m)}\right) u
\end{aligned}
$$




$$
\begin{aligned}
\dot{\zeta}_{1}^{(1)} & =\zeta_{2}^{(1)} \\
& \vdots \\
\dot{\zeta}_{r_{1}-1}^{(1)} & =\zeta_{r_{1}}^{(1)} \\
\dot{\zeta}_{r_{1}}^{(1)} & =W_{1}\left(\zeta^{(0)}, \zeta^{(1)}, \cdots, \zeta^{(m)}\right)+C_{1}\left(\zeta^{(0)}, \zeta^{(1)}, \cdots, \zeta^{(m)}\right) u \\
& \vdots \\
\dot{\zeta}_{1}^{(m)} & =\zeta_{2}^{(m)} \\
& \vdots \\
\dot{\zeta}_{r_{m}-1}^{(m)} & =\zeta_{r_{m}}^{(m)} \\
\dot{\zeta}_{r_{m}}^{(m)} & =W_{m}\left(\zeta^{(0)}, \zeta^{(1)}, \cdots, \zeta^{(m)}\right)+C_{m}\left(\zeta^{(0)}, \zeta^{(1)}, \cdots, \zeta^{(m)}\right) u \\
y_{1} & =\zeta_{1}^{(1)} \\
& \vdots \\
y_{m} & =\zeta_{1}^{(m)}
\end{aligned}
$$

where

$$
\begin{aligned}
& F_{1}\left(\zeta^{(0)}, \zeta^{(1)}, \cdots, \zeta^{(m)}\right) \\
& =\left[L_{f} t_{i}(x)\right]_{x=\Phi^{-1}(\zeta)}, \quad i=1, \cdots,\left(n-\sum_{i} r_{i}\right) \\
& G_{i}\left(\zeta^{(0)}, \zeta^{(1)}, \cdots, \zeta^{(m)}\right) \\
& =\left[L_{g} t_{i}(x)\right]_{x=\Phi^{-1}(\zeta)}, \quad i=1, \cdots,\left(n-\sum_{i} r_{i}\right) \\
& C_{i}\left(\zeta^{(0)}, \zeta^{(1)}, \cdots, \zeta^{(m)}\right) \\
& =\left[L_{g} L_{f}^{r_{i}-1} h_{i}(x)\right]_{x=\Phi^{-1}(\zeta)}, \quad i=1, \cdots, m \\
& W_{i}\left(\zeta^{(0)}, \zeta^{(1)}, \cdots, \zeta^{(m)}\right) \\
& =\left[L_{f}^{r_{i}} h_{i}(x)\right]_{x=\Phi^{-1}(s)}, \quad i=1, \cdots, m
\end{aligned}
$$

According to Isidori (1989), Eq. 7 represents a normal form for the nonlinear system described by Eq. 1, which is analogous to the one introduced for SISO nonlinear systems (Byrnes and Isidori, 1985, Kravaris, 1988). Its first $\left(n-\Sigma_{i} r_{i}\right)$ state equations form a nonlinear subsystem denoted by the superscript $(0)$, which will later be associated with the inverse dynamics and ultimately with the zero dynamics of the system. The remaining $\Sigma_{i} r_{i}$ state equations consist of $m$ subsystems, each one denoted by a superscript $(i)$, with $i=1, \cdots, m$. The $i$ th of these subsystems is of order $r_{i}$ and consists of a chain or $r_{i}-1$ pure integrations followed by a nonlinear state equation.

Remark 3. One obvious difference of the normal form of Eq. 7 from the SISO normal form arises from the fact that the zeroth nonlinear subsystem in Eq. 7 depends explicitly on the manipulated input vector $u$. Elimination of the manipulated input vector from this set of equations is possible only when the vector fields $g_{1}, \cdots, g_{m}$ form an involutive set, a condition that is trivially satisfied in the SISO case. In this case, the calculation of the appropriate scalar fields $t_{i}(x)$ would involve the solution of a set of partial differential equations (PDE), which is a nontrivial task.

We can now proceed and apply the inversion formula given in Theorem 1, Eq. 5, to the above normal form representation of the nonlinear system. The result will be the reduced inverse of the system, and is stated in Theorem 2; the proof is given in the Appendix.
Theorem 2. Consider a nonlinear system in the normal form of Eq. 7, and let

$\mathfrak{D}_{y_{1}}=\left[\begin{array}{c}y_{1} \\ \vdots \\ \frac{d^{r_{1}-1} y_{1}}{d t^{r_{1}-1}}\end{array}\right], \cdots, D_{y_{m}}=\left[\begin{array}{c}y_{m} \\ \vdots \\ \frac{d^{r_{m}-1} y_{m}}{d t^{r_{m}-1}}\end{array}\right], D^{*} \mathrm{y}=\left[\begin{array}{c}d^{r_{1} y_{1}} \\ d t^{r_{0}} \\ \vdots \\ \frac{d^{r_{m}} y_{m}}{d t^{\prime}}\end{array}\right]$

Then, the dynamic system:

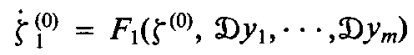

$$
\begin{aligned}
& +G_{1}\left(\zeta^{(0)}, D y_{1}, \cdots, D y_{m}\right) C\left(\zeta^{(0)}, D y_{1}, \cdots, D y_{m}\right)^{-1} \\
& \cdot\left[D^{*} y-W\left(\zeta^{(0)}, D D y_{1}, \cdots D D y_{m}\right)\right]
\end{aligned}
$$

$$
\begin{aligned}
& \dot{\zeta}_{n-\Sigma_{i} r_{i}}^{(0)}=F_{n-\Sigma_{i} r_{i}}\left(\zeta^{(0)}, D y_{1}, \cdots, D y_{m}\right) \\
& +G_{n-\Sigma_{i} r_{i}}\left(\zeta^{(0)}, D y_{1}, \cdots, D y_{m}\right) C\left(\zeta^{(0)}, D y_{1}, \cdots, D y_{m}\right)^{-1} \\
& \cdot\left[D^{*} y-W\left(\zeta^{(0)}, \mathfrak{D} y_{1}, \cdots, D y_{m}\right)\right]
\end{aligned}
$$

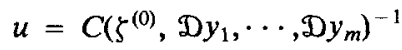

$$
\begin{aligned}
& \cdot\left[D^{*} y-W\left(\zeta^{(0)}, \mathfrak{D} y_{1}, \cdots, \mathfrak{D} y_{m}\right)\right]
\end{aligned}
$$

with

$$
W(\zeta)=\left[W_{1}(\zeta) \ldots W_{m}(\zeta)\right]^{T} \text { and } C(\zeta)=\left[C_{1}(\zeta) \ldots C_{m}(\zeta)\right]^{T}
$$

defined in Eq. 8, represents the reduced inverse of the original system.

Remark 4. In the case of a SISO nonlinear sy with relative order $r$, the reduced inverse given $b$ Eq. 10 takes the form:

$$
\begin{aligned}
& \dot{\zeta}_{1}^{(0)}=F_{1}\left(\zeta^{(0)}, y, \frac{d y}{d t}, \cdots, \frac{d^{r-1} y}{d t^{r-1}}\right) \\
& +G_{1}\left(\zeta^{(0)}, y, \frac{d y}{d t}, \cdots, \frac{d^{r-1} y}{d t^{r-1}}\right) C\left(\zeta^{(0)}, y, \frac{d y}{d t}, \cdots, \frac{d^{r-1} y}{d t^{r-1}}\right)^{-1} \\
& \times\left[\frac{d^{r} y}{d t^{r}}-W\left(\zeta^{(0)}, y, \frac{d y}{d t}, \cdots, \frac{d^{r-1} y}{d t^{r-1}}\right)\right] \\
& \dot{\zeta}_{n-r}^{(0)}=F_{n-r}\left(\zeta^{(0)}, y, \frac{d y}{d t}, \cdots, \frac{d^{r-1} y}{d t^{r-1}}\right) \\
& +G_{n-r}\left(\zeta^{(0)}, y, \frac{d y}{d t}, \cdots, \frac{d^{r-1} y}{d t^{r-1}}\right) C\left(\zeta^{(0)}, y, \frac{d y}{d t}, \cdots \cdot \frac{d^{r-1} y}{d t^{r-1}}\right)^{-1} \\
& \times\left[\frac{d^{r} y}{d t^{r}}-W\left(\zeta^{(0)}, y, \frac{d y}{d t}, \cdots, \frac{d^{r-1} y}{d t^{r-1}}\right)\right] \\
& u=C\left(\zeta^{(0)}, y, \frac{d y}{d t}, \cdots, \frac{d^{r-1} y}{d t^{r-1}}\right)^{-1} \\
& \times\left[\frac{d^{r} y}{d t^{r}}-W\left(\zeta^{(0)}, y, \frac{d y}{d t}, \cdots, \frac{d^{r-1} y}{d t^{r-1}}\right)\right]
\end{aligned}
$$


where

$$
\begin{aligned}
& C\left(\zeta^{(0)}, y, \frac{d y}{d t}, \cdots, \frac{d^{r-1} y}{d t^{r-1}}\right)=L_{g} L_{f}^{r-1} h\left(\zeta^{(0)}, y, \frac{d y}{d t}, \cdots, \frac{d^{r-1} y}{d t^{r-1}}\right) \\
& W\left(\zeta^{(0)}, y, \frac{d y}{d t}, \cdots, \frac{d^{r-1} y}{d t^{r-1}}\right)=L_{f}^{r} h\left(\zeta^{(0)}, y, \frac{d y}{d t}, \cdots, \frac{d^{r-1} y}{d t^{r-1}}\right)
\end{aligned}
$$

One can always choose the scalar fields $t_{1}(x), \cdots, t_{n-r}(x)$ so that $G_{i}(\zeta)=0, i=1, \cdots, m$, in which case we obtain exactly the realization of the minimal-order inverse originally obtained for SISO systems (Kravaris, 1988).

Remark 5. The order of the realization of Eq. 10 is $\left(n-\Sigma_{i} r_{i}\right)$ and its minimality is a consequence of the special structure of the normal form, which incorporates explicitly the outputs of the system and their derivatives as part of the coordinate system.

Up to this point, we have been able to generalize the SISO results obtained for the full-order and the reduced inverse in a MIMO context. The next step is the appropriate definition of a concept of MIMO forced zero dynamics. This will allow a complete characterization of the stability of the inverse.

\section{Zero Dynamics in Nonlinear Multivariable Systems}

The concept of zeros in MIMO linear systems has been a subject of investigation during the last twenty years (e.g., MacFarlane and Karcanias, 1976; Holt and Morari, 1985). It is now understood that a meaningful characterization of zeros in a MIMO setting goes beyond the zeros of the individual transfer functions between the inputs and outputs; it involves the concept of transmission zeros, which can be defined as the poles of the inverse system. The terminology "transmission zeros" is due to the transmission blocking property of the zeros $[y(t)=0$ for every $t]$, under an appropriate input and an appropriate set of initial conditions.

In what follows, a nonlinear analog of the notion of transmission zeros is rigorously established, through the concept of MIMO zero dynamics.

Definition 3 (Isidori and Moog, 1988). Consider a MIMO nonlinear system of the form of Eq. 1 with nonsingular characteristic matrix. The (unforced) zero dynamics of the system of Eq. 1 is the dynamics of its (unforced) reduced inverse.

In general, the algorithm of Isidori and Moog for the construction of the reduced inverse can be followed for the calculation of the zero dynamics. For systems with nonsingular characteristic matrix, however, the explicit formula for the reduced inverse developed in Theorem 2 facilitates the calculation of an explicit formula for the unforced zero dynamics. This is obtained by simply setting the outputs and their derivatives in Eq. 10 equal to 0 . The resulting system takes the form:

$$
\begin{aligned}
\dot{\zeta}_{1}^{(0)}= & F_{1}\left(\zeta^{(0)}, 0, \cdots, 0\right)-G_{1}\left(\zeta^{(0)}, 0, \cdots, 0\right) \\
& \quad \times C\left(\zeta^{(0)}, 0, \cdots, 0\right)^{-1} W\left(\zeta^{(0)}, 0, \cdots, 0\right) \\
\dot{\zeta}_{n-\Sigma_{i} r_{i}}^{(0)} & \\
& \\
& \quad F_{n-\Sigma_{i} r_{i}}\left(\zeta^{(0)}, 0, \cdots, 0\right)-G_{n-\Sigma_{i} r_{i}}\left(\zeta^{(0)}, 0, \cdots, 0\right) \\
& \times C\left(\zeta^{(0)}, 0, \cdots, 0\right)^{-1} W\left(\zeta^{(0)}, 0, \cdots, 0\right)
\end{aligned}
$$

Remark 6. The transmission blocking property of the linear transmission zeros generalizes in a nonlinear context as well (Isidori and Moog, 1988), in the sense that the above (unforced) zero dynamics is identical to the zero-output constrained dynamics, that is, the internal dynamics of the system when the output is forced to remain zero for all times.

Remark 7. If the concept of zero dynamics is meaningful, one would expect that in the vicinity of an equilibrium point it should give the same information as the zeros of the linear approximation of the original nonlinear system. In fact, D'Andrea and Praly (1988) have shown that the eigenvalues of the linearized zero dynamics coincide with the zeros of the linearized system. Furthermore, in the case of linear systems the zero dynamics is a linear dynamic system with poles at the process zeros.

Once we have obtained a meaningful generalization of the notion of transmission zeros, we can also obtain a characterization of minimum-phase behavior in a MIMO nonlinear setting. In particular, the following definition is due to Byrnes and Isidori (1988).

Definition 4. The nonlinear system in the form of Eq. 7 is minimum-phase if its (unforced) zero dynamics, Eq. 11, is asymptotically stable. It is nonminimum-phase if its (unforced) zero dynamics is unstable.

The above characterization of nonminimum-phase behavior is in complete analogy with the linear results; the difference is that instead of depending on the location of the transmission zeros in the complex plane, minimum-phase behavior depends on the stability of the zero dynamics. It is important to note, however, that the characterization of minimum-phase behavior of definition 4 is only concerned with the asymptotic stability characteristics of the (unforced) zero dynamics. A complete stability characterization of the zero dynamics should also take into account input/state stability considerations. For this reason, we introduce here a concept of forced zero dynamics, as follows:

Definition 5. For a MIMO nonlinear system in the normal form of Eq. 7, the dynamic system

$$
\begin{aligned}
\dot{\zeta}_{1}^{(0)}= & F_{1}\left(\zeta^{(0)}, \mathcal{u}_{1}, \cdots, \mathcal{u}_{m}\right) \\
& +G_{1}\left(\zeta^{(0)}, \mathcal{u}_{1}, \cdots, \mathcal{u}_{m}\right) C\left(\zeta^{(0)}, \mathcal{u}_{1}, \cdots, u_{m}\right)^{-1} \\
& \quad \times\left[\mathcal{u}_{*}-W\left(\zeta^{(0)}, \mathcal{u}_{1}, \cdots, u_{m}\right)\right] \\
\vdots & \quad \\
\dot{\zeta}_{n-\Sigma_{i} r_{i}}^{(0)}= & F_{n-\Sigma_{i} r_{i}}\left(\zeta^{(0)}, \mathcal{u}_{1}, \cdots, \mathcal{u}_{m}\right) \\
+ & G_{n-\Sigma_{i} r_{i}}\left(\zeta^{(0)}, \mathcal{u}_{1}, \cdots, \mathcal{u}_{m}\right) C\left(\zeta^{(0)}, \mathcal{u}_{1}, \cdots, \mathcal{u}_{m}\right)^{-1} \\
& \quad \times\left[\mathcal{u}_{*}-W\left(\zeta^{(0)}, \mathcal{u}_{1}, \cdots, \mathcal{u}_{m}\right)\right]
\end{aligned}
$$

with inputs $\mathcal{U}_{i} \in \mathbb{R}^{r_{i}}$ and $\mathcal{U}_{*} \in \mathbb{R}^{m}$, is called the forced zero dynamics.

The above dynamic system is nothing more than the reduced inverse of the original system, with the outputs and their derivatives viewed as forcing inputs. A variety of stability concepts can be defined for the above forced zero dynamics; in general, perturbations in the initial conditions as well as changes in its inputs can be considered. Alternative stability concepts can then lead to alternative definitions of minimum-phase behavior, depending on the context. 


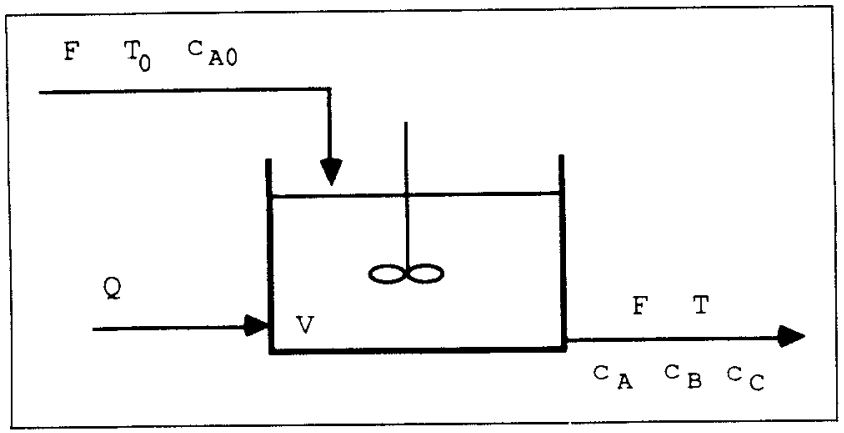

Figure 1. A CSTR.

\section{Illustrative Examples}

We now illustrate the calculation of the unforced and forced zero dynamics in two chemical engineering examples.

\section{Example 1}

Consider a continuous stirred tank reactor (CSTR), Figure 1 , in which the series/parallel Van de Vusse reaction is taking place (Van de Vusse, 1964):

$$
\begin{gathered}
A \rightarrow B \rightarrow C \\
2 A \rightarrow D
\end{gathered}
$$

The rates of formation of $A$ and $B$ are assumed to be:

$$
\begin{aligned}
& r_{A}=-k_{1} C_{A}-k_{3} C_{A}^{2} \\
& r_{B}=k_{1} C_{A}-k_{2} C_{B}
\end{aligned}
$$

where

$$
k_{i}=Z_{i} \exp \left(-\frac{E_{i}}{R T}\right)
$$

The mass balances for $A$ and $B$ and the energy balance are given by:

$$
\begin{aligned}
V \frac{d C_{A}}{d t}=F\left(C_{A 0}-C_{A}\right)+V\left(-k_{1} C_{A}-k_{3} C_{A}^{2}\right) \\
V \frac{d C_{B}}{d t}=F\left(-C_{B}\right)+V\left(k_{1} C_{A}-k_{2} C_{B}\right) \\
V \rho c_{p} \frac{d T}{d t}=F \rho c_{p}\left(T_{0}-T\right)+V\left[\left(-\Delta H_{1}\right) k_{1} C_{A}+\left(-\Delta H_{2}\right) k_{2} C_{B}\right. \\
\left.+\left(-\Delta H_{3}\right) k_{3} C_{A}^{2}\right]+Q
\end{aligned}
$$

We wish to maintain $C_{B}$ and $T$ at their set points, by manipulating the dilution rate $F / V$ and the heat input $Q$. By letting:

$$
x_{1}=C_{A}, x_{2}=C_{B}, x_{3}=T
$$

and

$$
u_{1}=\frac{F}{V}, u_{2}=Q
$$

$$
y_{1}=C_{B}, y_{2}=T
$$

the system is put in the form of Eq. 1, with:

$$
\begin{aligned}
& f(x)= \\
& {\left[\begin{array}{c}
-k_{1}\left(x_{3}\right) x_{1}-k_{3}\left(x_{3}\right) x_{1}^{2} \\
k_{1}\left(x_{3}\right) x_{1}-k_{2}\left(x_{3}\right) x_{2} \\
\frac{1}{\rho c_{p}}\left[\left(-\Delta H_{1}\right) k_{1}\left(x_{3}\right) x_{1}+\left(-\Delta H_{2}\right) k_{2}\left(x_{3}\right) x_{2}+(-\Delta H) k_{3}\left(x_{3}\right) x_{1}^{2}\right]
\end{array}\right]} \\
& g_{1}(x)=\left[\begin{array}{c}
C_{A 0}-x_{1} \\
-x_{2} \\
\frac{T_{0}-x_{3}}{\rho c_{p}}
\end{array}\right], g_{2}(x)=\left[\begin{array}{c}
0 \\
0 \\
\frac{1}{V \rho c_{p}}
\end{array}\right] \\
& h_{1}(x)=x_{2}, h_{2}(x)=x_{3}
\end{aligned}
$$

Applying definition 1 for the calculation of the relative orders, we find:

$$
\begin{gathered}
L_{g_{1}} h_{1}=-x_{2} \\
L_{g_{1}} h_{2}=\frac{T_{0}-x_{3}}{\rho c_{p}} \\
L_{g_{2}} h_{1}=0 \\
L_{g_{2}} h_{2}=\frac{1}{V \rho c_{p}}
\end{gathered}
$$

Consequently, the relative orders of $r_{1}=1$ and $r_{2}=1$. Moreover, the characteristic matrix

$$
C(x)=\left[\begin{array}{ll}
L_{g_{1}} h_{1} & L_{g_{2}} h_{1} \\
L_{g_{1}} h_{2} & L_{g_{2}} h_{2}
\end{array}\right]=\left[\begin{array}{ll}
-x_{2} & 0 \\
\frac{T_{0}-x_{3}}{\rho c_{p}} & \frac{1}{V \rho c_{p}}
\end{array}\right]
$$

is generically nonsingular, since

$$
\operatorname{det} C(x)=\frac{-x_{2}}{V \rho c_{p}} \not \equiv
$$

The next step toward the calculation of the zero dynamics is the transformation of the system to the normal form, Eq. 7. According to Eq. 6, we are seeking an invertible coordinate transformation of the form:

$$
\zeta=\left[\begin{array}{c}
\zeta_{1}^{(0)} \\
-- \\
\zeta_{1}^{(1)} \\
-\overline{\zeta_{1}^{(2)}}
\end{array}\right]=\left[\begin{array}{c}
t_{1}(x) \\
--- \\
h_{1}(x) \\
--- \\
h_{2}(x)
\end{array}\right]=\left[\begin{array}{c}
t_{1}(x) \\
-- \\
x_{2} \\
--- \\
x_{3}
\end{array}\right]
$$

An obvious choice for the scalar field $t_{1}(x)$ is $t_{1}(x)=x_{1}$. Consequently, the system in the original coordinate system $x$ is already in the normal form. Moreover, the manipulated input vector $u$ does not appear in the zeroth dynamical subsystem. A direct calculation of the reduced inverse system, according to Eq. 10, yields: 


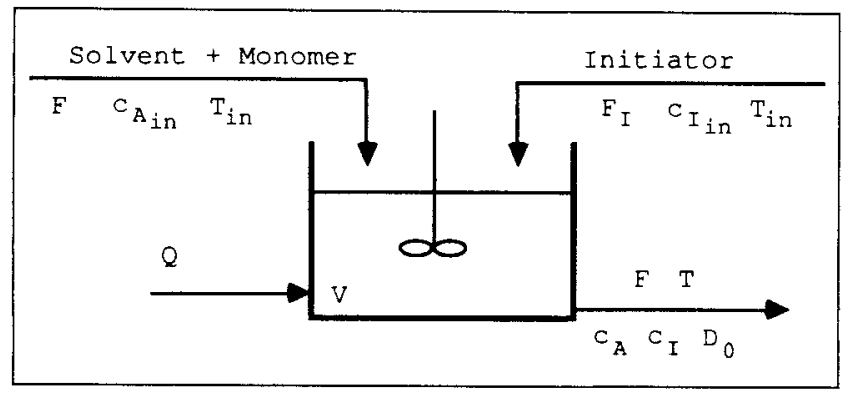

Figure 2. A polymerization CSTR.

$$
\begin{aligned}
\dot{\zeta}_{1}^{(0)}=-Z_{1} \exp \left(-\frac{E_{1}}{R y_{2}}\right) \zeta_{1}^{(0)}-Z_{3} \exp \left(-\frac{E_{3}}{R y_{2}}\right)\left(\zeta_{1}^{(0)}\right)^{2} \\
+\left(C_{A 0}-\zeta_{1}^{(0)}\right) \frac{Z_{1} \exp \left(-\frac{E_{1}}{R y_{2}}\right) \zeta_{1}^{(0)}-Z_{2} \exp \left(-\frac{E_{2}}{R y_{2}}\right) y_{1}}{y_{2}}
\end{aligned}
$$

where the $\zeta$ notation is used to conform with the notation in Eq. 10.

The above system also represents the forced zero dynamics, Eq. 12, with forcing inputs $u_{1}=y_{1}$ and $u_{2}=y_{2}$. Finally, substituting $y_{1}$ and $y_{2}$ by their steady state values $y_{1 s}$ and $y_{2 s}$, we obtain the unforced zero dynamics:

$$
\begin{aligned}
\dot{\zeta}_{1}^{(0)}=- & Z_{1} \exp \left(-\frac{E_{1}}{R y_{2 s}}\right) \zeta_{1}^{(0)}-Z_{3} \exp \left(-\frac{E_{3}}{R y_{2 s}}\right)\left(\zeta_{1}^{(0)}\right)^{2} \\
+\left(C_{A 0}-\zeta_{1}^{(0)}\right) & \frac{Z_{1} \exp \left(-\frac{E_{1}}{R y_{2 s}}\right) \zeta_{1}^{(0)}-Z_{2} \exp \left(-\frac{E_{2}}{R y_{2 s}}\right) y_{1 s}}{y_{2 s}}
\end{aligned}
$$

\section{Example 2}

Consider a CSTR, Figure 2, where free-radical polymerization of methylmethacrylate takes place, with azo-bisisobutyronitrile as initiator and toluene as solvent. Considering the standard mechanism of the free-radical polymerization and under the assumptions of:

- Perfect mixing

- No gel effect, because of low conversion of the monomer

- Constant density and heat capacity of the reacting mixture

- No polymer in the inlet streams

- Constant reactor volume (constant total volumetric flow rate of streams)

- Negligible flow rate of the initiator solution in comparison with the flow rate of monomer solution

- Neglected inhibition and chain transfer to solvent reaction

- Quasisteady-state and long-chain hypothesis

we obtain the following mass and energy balances (e.g., Daoutidis et al., 1990):

$$
\begin{aligned}
\frac{d C_{A}}{d t} & =-\left(k_{P}+k_{f_{a}}\right) C_{A} P_{0}\left(C_{I}, T\right)+\frac{F\left(C_{A_{i n}}-C_{A}\right)}{V} \\
\frac{d C_{I}}{d t} & =-k_{I} C_{I}+\frac{F_{I} C_{I_{i n}}-F C_{I}}{V}
\end{aligned}
$$

$$
\begin{aligned}
\frac{d T}{d t} & =k_{P} C_{A} \frac{\left(-\Delta H_{P}\right)}{\rho C_{p}} P_{0}\left(C_{I}, T\right)+\frac{Q}{V \rho c_{p}}+\frac{F\left(T_{i n}-T\right)}{V} \\
\frac{d D_{0}}{d t} & =\left(0.5 k_{T_{c}}+k_{T_{d}}\right)\left[P_{0}\left(C_{I}, T\right)\right]^{2}+k_{f_{a}} C_{A} P_{0}\left(C_{I}, T\right)-\frac{F D_{0}}{V}
\end{aligned}
$$

where

$$
P_{0}\left(C_{I}, T\right)=\left(\frac{2 \phi k_{I} C_{I}}{k_{T_{d}}+k_{T_{\mathrm{c}}}}\right)^{0.5}
$$

and

$$
k_{i}=Z_{i} \exp \left(-\frac{E_{i}}{R T}\right)
$$

We consider control of the temperature of the polymer product $T$ and the molar concentration of the dead polymer chains $D_{0}$, by manipulating the heat input to the reactor $Q$ and the volumetric flow rate of the initiator $F_{I}$. Thus, following the standard procedure and letting

$$
x_{1}=C_{A}, x_{2}=C_{I}, x_{3}=T, x_{4}=D_{0}
$$

and

$$
\begin{aligned}
& u_{1}=F_{1}, u_{2}=Q \\
& y_{1}=T, y_{2}=D_{0}
\end{aligned}
$$

the system dynamic equations are put in the form of Eq. 1, where

$$
\begin{aligned}
& f(x)=\left[\begin{array}{l}
f_{1}(x) \\
f_{2}(x) \\
f_{3}(x) \\
f_{4}(x)
\end{array}\right]= {\left[\begin{array}{c}
-\left(k_{P}+k_{f_{e}}\right) x_{1} P_{0}+\frac{F\left(C_{A_{\text {in }}}-x_{1}\right)}{V} \\
-k_{I} x_{2}-\frac{F x_{2}}{V} \\
k_{P} x_{1} \frac{\left(-\Delta H_{P}\right)}{\rho c_{p}} P_{0}+\frac{F\left(T_{i n}-x_{3}\right)}{V} \\
\left(0.5 k_{T_{c}}+k_{T_{d}}\right) P_{0}^{2}+k_{f_{d}} x_{1} P_{0}-\frac{F x_{4}}{V}
\end{array}\right] } \\
& g_{1}(x)= {\left[\begin{array}{c}
0 \\
\frac{C_{I_{i n}}}{V} \\
0 \\
0
\end{array}\right], g_{2}(x)=\left[\begin{array}{c}
0 \\
0 \\
\frac{1}{V \rho c_{p}} \\
0
\end{array}\right] } \\
& h_{1}(x)=x_{3}, h_{2}(x)=x_{4}
\end{aligned}
$$

Following definition 1 for the calculation of the relative orders, we find:

$$
\begin{gathered}
L_{g_{1}} h_{1}=0 \\
L_{g_{2}} h_{1}=\frac{1}{V \rho c_{p}} \\
L_{g_{1}} h_{2}=0 \\
L_{g_{2}} h_{2}=0
\end{gathered}
$$




$$
\begin{gathered}
L_{g_{1}} L_{f} h_{2}=\frac{\partial f_{4}}{\partial x_{2}} \frac{C_{I_{i n}}}{V} \\
L_{g_{2}} L_{f} h_{2}=\frac{\partial f_{4}}{\partial x_{3}} \frac{1}{V \rho c_{p}}
\end{gathered}
$$

Consequently, the relative orders are $r_{1}=1$ and $r_{2}=2$. Moreover, the characteristic matrix

$$
C(x)=\left[\begin{array}{lr}
L_{g_{1}} h_{1}(x) & L_{g_{2}} h_{1}(x) \\
L_{g_{1}} L_{f} h_{2}(x) & L_{g_{2}} L_{f} h_{2}(x)
\end{array}\right]=\left[\begin{array}{lr}
0 & \frac{1}{V \rho c_{p}} \\
\frac{\partial f_{4}}{\partial x_{2}} \frac{C_{I_{i n}}}{V} & \frac{\partial f_{4}}{\partial x_{3}} \frac{1}{V \rho c_{p}}
\end{array}\right]
$$

is generically nonsingular.

It can be easily verified that the coordinate transformation:

$$
\zeta=\left[\begin{array}{c}
\zeta_{1}^{(0)} \\
- \\
\zeta_{1}^{(1)} \\
-- \\
\zeta_{1}^{(2)} \\
\zeta_{2}^{(2)}
\end{array}\right]=\left[\begin{array}{c}
t_{1}(x) \\
--- \\
h_{1}(x) \\
-- \\
h_{2}(x) \\
L_{f} h_{2}(x)
\end{array}\right]=\left[\begin{array}{c}
x_{2} \\
---- \\
x_{3} \\
-x_{4} \\
f_{4}\left(x_{1}, x_{2}, x_{3}, x_{4}\right)
\end{array}\right]
$$

is invertible. Therefore, it can be used to transform the system in its normal form, obtaining:

$$
\begin{aligned}
\dot{\zeta}_{1}^{(0)} & =F_{1}(\zeta)+G_{1}(\zeta) u \\
\dot{\zeta}_{1}^{(1)} & =W_{1}(\zeta)+C_{1}(\zeta) u \\
\dot{\zeta}_{1}^{(2)} & =\zeta_{2}^{(2)} \\
\dot{\zeta}_{2}^{(2)} & =W_{2}(\zeta)+C_{2}(\zeta) u \\
y_{1} & =\zeta_{1}^{(1)} \\
y_{2} & =\zeta_{1}^{(2)}
\end{aligned}
$$

where

$$
\begin{aligned}
& F_{1}(\zeta)=L_{f} t_{1}=f_{2} \\
& G_{1}(\zeta)=L_{g} t_{1}=\left[\begin{array}{ll}
\frac{C_{I_{i n}}}{V} & 0
\end{array}\right] \\
& C_{1}(\zeta)=L_{g} h_{1}=\left[\begin{array}{ll}
0 & \frac{1}{V \rho c_{p}}
\end{array}\right] \\
& W_{1}(\zeta)=L_{f} h_{1}=f_{3} \\
& C_{2}(\zeta)=L_{g} L_{f} h_{2}=\left[\begin{array}{lll}
\frac{\partial f_{4}}{\partial x_{2}} \frac{C_{I_{m}}}{V} \frac{\partial f_{4}}{\partial x_{3}} \frac{1}{V \rho c_{p}}
\end{array}\right] \\
& W_{2}(\zeta)=L_{f}^{2} h_{2} \quad=L_{f} f_{4}=\sum_{i=1}^{4} \frac{\partial f_{4}}{\partial x_{i}} f_{i}
\end{aligned}
$$

and the righthand-side expressions in the above equations are evaluated in terms of the $\zeta$ coordinates. Similarly to example 1 , one can calculate the forced and unforced zero dynamics in a straightforward way. Calculation of the unforced zero dynamics, Eq. 11, yields the following dynamic system:

$$
\dot{\zeta}_{1}^{(0)}=f_{2}\left(\zeta_{1}^{(0)}, \zeta_{s}^{(1)}, \zeta_{s}^{(2)}\right)+\left\{\begin{array}{l}
\frac{\partial f_{4}}{\partial x_{3}}\left(\zeta_{1}^{(0)}, \zeta_{s}^{(1)}, \zeta_{s}^{(2)}\right) \\
\frac{\partial f_{4}}{\partial x_{2}}\left(\zeta_{1}^{(0)}, \zeta_{s}^{(1)}, \zeta_{s}^{(2)}\right)
\end{array}\right.
$$

$$
\left.\times f_{3}\left(\zeta_{1}^{(0)}, \zeta_{s}^{(1)}, \zeta_{s}^{(2)}\right)-\frac{1}{\frac{\partial f_{4}}{\partial x_{2}}\left(\zeta_{1}^{(0)}, \zeta_{s}^{(1)}, \zeta_{s}^{(2)}\right)} L_{f} f_{4}\left(\zeta_{1}^{(1)}, \zeta_{s}^{(1)}, \zeta_{s}^{(2)}\right)\right\}
$$

\section{Role of Zero Dynamics in Input/Output Linearization}

The previous sections were devoted to establishing a sound conceptual framework, in order to address the issue of internal stability under input/output linearizing state feed back. In what follows, we briefly review input/output linearization for MIMO nonlinear systems, we provide an interpretation of input/output linearization as canceling the forced zero dynamics of the system and, finally, we develop precise internal stability conditions for the closed-loop system.

\section{Input/output linearization}

Consider a general MIMO nonlinear system described by Eq. 1, with finite relative orders $r_{i}$ and nonsingular characteristic matrix $C(x)$. For this system, the problem of input/ output linearization is that of calculating a static state feedback control law that results in a prespecified linear input/output closed-loop behavior. Kravaris and Soroush (1990) showed that the control law:

$$
u=\left\{\left[\beta_{1 r_{1}} \cdots \beta_{m r_{m}}\right] C(x)\right\}^{-1}\left\{v-\sum_{i=1}^{m} \sum_{k=0}^{r_{i}} \beta_{i k} L_{f}^{k} h_{i}(x)\right\}
$$

induces a linear closed-loop input/output behavior of the form:

$$
\sum_{i=1}^{m} \sum_{k=0}^{r_{i}} \beta_{i k} \frac{d^{k} y_{i}}{d t^{k}}=v
$$

where $\beta_{i k}$ is an $m$ vector of adjustable constant parameters with

$$
\operatorname{det}\left[\beta_{1 r_{1}} \beta_{2 r_{2}} \cdots \beta_{m r_{m}}\right] \neq 0,
$$

and $v$ is an $m$ vector of reference inputs. Under the above control law, the linear closed-loop system does not have any finite zeros. On the other hand, the poles of the closed-loop system are the roots of the characteristic equation:

$$
\operatorname{det}\left[\left(\sum_{k=0}^{r_{1}} \beta_{1 k} s^{k}\right)\left(\sum_{k=0}^{r_{2}} \beta_{2 k} s^{k}\right) \cdots\left(\sum_{k=0}^{r_{m}} \beta_{m k} s^{k}\right)\right]=0 .
$$

Clearly, the order of the closed-loop system is $r_{1}+r_{2}+\cdots+r_{m}$ and its bounded-input/bounded-output (BIBO) stability characteristics depend on the choice of the adjustable parameters $\beta_{i k}$. In addition to BIBO stability, the closed-loop system must 
also be internally asymptotically stable, in the sense that any perturbations in the initial conditions of the state variables in the unforced closed-loop system must die out. Some insight on this issue can be obtained as follows: Recall that, in general, $r_{1}+r_{2}+\cdots+r_{m} \leq n$. This implies that the state feedback of Eq. 13 results in some reduction of the order of the original system. In other words, some dynamics [of order $n-\left(r_{1}+\right.$ $\left.\left.r_{2}+\cdots+r_{m}\right)\right]$ is canceled. One would therefore intuitively expect that the internal stability of the closed-loop system should be closely related to the stability of the canceled dynamics. In what follows, we show that the canceled dynamics is exactly the forced zero dynamics of the system and we also provide precise internal stability conditions for the closed-loop system.

\section{Input/output linearizing feedback cancels the forced zero dynamics}

For convenience, consider the original nonlinear system, transformed in the normal form of $\mathrm{Eq}$. 7. In the new coordinates, the input/output linearizing control law given by Eq. 13 takes the form:

$$
\begin{aligned}
& u=\left\{\left[\beta_{1 r_{1}} \cdots \beta_{m r_{m}}\right] C(\zeta)\right\}^{-1} \\
&\left.\times\left\{v-\sum_{i=1}^{m} \sum_{k=0}^{\left(r_{i}-1\right)} \beta_{i k}\right\}_{k+1}^{(i)}-\left[\beta_{1 r_{1}} \cdots \beta_{m r_{m}}\right] W(\zeta)\right\}
\end{aligned}
$$

Substituting the above control law in Eq. 7, we obtain the following closed-loop dynamics:

$$
\begin{aligned}
& \dot{\zeta}_{1}^{(0)}=F_{1}(\zeta)+G_{1}(\zeta) C(\zeta)^{-1} \\
& \left\{\left[\beta_{1 r_{1}} \cdots \beta_{m r_{m}}\right]^{-1}\left(v-\sum_{i=1}^{m} \sum_{k=0}^{\left(r_{i}-1\right)} \beta_{i k} \zeta_{k+1}^{(i)}\right)-W(\zeta)\right\} \\
& \dot{\zeta}_{n-\Sigma_{i} r_{i}}^{(0)}=F_{n-\Sigma_{i} r_{i}}(\zeta)+G_{n-\Sigma_{i} r_{i}}(\zeta) C(\zeta)^{-1} \\
& \left\{\left[\beta_{1 r_{1}} \cdots \beta_{m r_{m}}\right]^{-1}\left(v-\sum_{i=1}^{m} \sum_{k=0}^{\left(r_{i}-1\right)} \beta_{i k} \zeta_{k+1}^{(i)}\right)-W(\zeta)\right\} \\
& \dot{\zeta}_{1}^{(1)}=\zeta_{2}^{(1)} \\
& \dot{\zeta}_{r_{1}-1}^{(1)}=\zeta_{r_{1}}^{(1)}
\end{aligned}
$$

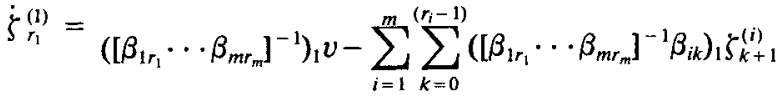

$$
\begin{aligned}
& \dot{\zeta}_{1}^{(m)}=\zeta_{2}^{(m)} \\
& \text { : } \\
& \dot{\zeta}_{r_{m}-1}^{(m)}=\zeta_{r_{m}}^{(m)} \\
& \dot{\zeta}_{r_{m}}^{(m)}=\left(\left[\beta_{1 r_{1}} \cdots \beta_{m r_{m}}\right]^{-1}\right)_{m} v-\sum_{i=1}^{m} \sum_{k=0}^{\left(r_{i}-1\right)}\left(\left[\beta_{1 r_{1}} \cdots \beta_{m r_{m}}\right]^{-1} \beta_{i k}\right)_{m} \zeta_{k+1}^{(i)} \\
& y_{1}=\zeta_{1}^{(1)} \\
& y_{m}=\zeta_{i}^{(m)}
\end{aligned}
$$

where the symbol ( $)_{i}$ denotes the $i$ th row of a matrix.

Clearly, the input/output linearizing control law given by Eq. 16 makes the first $\left(n-\Sigma_{i} r_{i}\right)$ state variables $\zeta_{1}^{(0)}, \cdots, \zeta_{n-\Sigma_{r_{i}}}^{(0)}$ unobservable in the closed-loop system, since they only appear in the zeroth subsystem and not in the remaining dynamics that determines the closed-loop input/output response. On the other hand, the unobservable dynamics of the $\zeta^{(0)}$ state variables is exactly the forced zero dynamics, Eq. 12, with inputs

$$
\mathfrak{u}_{1}=\zeta^{(1)}, \cdots, \mathfrak{u}_{m}=\zeta^{(m)}
$$

and

$$
\mathcal{U}_{*}=\left[\beta_{1 r_{1}} \cdots \beta_{m r_{m}}\right]^{-1}\left(v-\sum_{i=1}^{m} \sum_{k=0}^{\left(r_{i}-1\right)} \beta_{i k} \zeta_{k+1}^{(i)}\right)
$$

In the above sense, input/output linearizing state feedback cancels the forced zero dynamics of the system. The above conclusion generalizes a similar result obtained in a SISO context (Kravaris, 1988) and leads very naturally to the derivation of internal stability conditions for the closed-loop system.

\section{Internal stability under input/output linearizing feed- back}

Consider the closed-loop system under input/output linearizing feedback in the normal form coordinates, Eq. 17. The unforced closed-loop system is obtained by simply letting $v=$ 0 , and takes the form:

$$
\begin{aligned}
& \dot{\zeta}_{1}^{(0)}=F_{1}(\zeta)+G_{1}(\zeta) C(\zeta)^{-1} \\
& \times\left\{-\sum_{i=1}^{m} \sum_{k=0}^{\left(r_{i}-1\right)}\left[\beta_{1 r_{1}} \cdots \beta_{m r_{m}}\right]^{-1} \beta_{i k} \zeta_{k+1}^{(i)}-W(\zeta)\right\} \\
& \dot{\zeta}_{n-\Sigma_{i} r_{i}}^{(0)}=F_{n-\Sigma_{i} r_{i}}(\zeta)+G_{n-\Sigma_{i} r_{i}}(\zeta) C(\zeta)^{-1} \\
& \times\left\{-\sum_{i=1}^{m} \sum_{k=0}^{\left(r_{i}-1\right)}\left[\beta_{1 r_{1}} \cdots \beta_{m r_{m}}\right]^{-1} \beta_{i k} \zeta_{k+1}^{(i)}-W(\zeta)\right\} \\
& \dot{\zeta}_{1}^{(1)}=\zeta_{2}^{(1)} \\
& \dot{\zeta}_{r_{1}-1}^{(1)}=\zeta_{r_{1}}^{(1)} \\
& \dot{\zeta}_{r_{1}}^{(1)}=-\sum_{i=1}^{m} \sum_{k=0}^{\left(r_{i}-1\right)}\left(\left[\beta_{1 r_{1}} \cdots \beta_{m r_{m}}\right]^{-1} \beta_{i k}\right)_{1} \zeta_{k+1}^{(i)} \\
& \dot{\zeta}_{1}^{(m)}=\zeta_{2}^{(m)} \\
& \vdots \\
& \dot{\zeta}_{r_{m}-1}^{(m)}=\zeta_{r_{m}}^{(m)} \\
& \dot{\zeta}_{r_{m}}^{(m)}=-\sum_{i=1}^{m} \sum_{k=0}^{\left(r_{i}-1\right)}\left(\left[\beta_{1 r_{1}} \cdots \beta_{m r_{m}}\right]^{-1} \beta_{i k}\right)_{m} \zeta_{k+1}^{(i)} \\
& y_{1}=\zeta_{1}^{(1)} \\
& y_{m}=\zeta_{1}^{(m)}
\end{aligned}
$$


Note that if BIBO stability is enforced in the closed-loop system by appropriate choice of $\beta_{i k}$, then any deviations from the equilibrium in the initial conditions of the state variables will generate exponentially decaying signals for the output and its derivatives, the states $\zeta^{(1)}, \cdots, \zeta^{(m)}$. These signals will in turn be inputs to the forced zero dynamics. Clearly, the asymptotic stability characteristics of the above closed-loop system will then depend on the input/state stability characteristics of the forced zero dynamics. The above arguments lead to the following theorem:

Theorem 3. Consider the nonlinear system in the form of Eq. 7, under the control law given by Eq. 16. Then, the closedloop system will be internally asymptotically stable if the forced zero dynamics is stable, in the sense that for any set of initial conditions $\zeta^{(0)}(0)$ and any exponential decaying inputs $u_{1}, \cdots, u_{m}, u_{*}$ :

$$
\lim _{t \rightarrow \infty} \zeta_{i}^{(0)}(t)=0, \quad i=1, \cdots,\left(n-\Sigma_{i} r_{i}\right)
$$

The above theorem provides a precise characterization of internal stability in the large, under input/output linearizing state feedback. In analogy with the SISO case, it is the inherent characteristics of the nonlinear process, and in particular the stability characteristics of its forced zero dynamics, that determine the assessment of internal stability in the closed loop.

It is important to note that the stability condition imposed on the forced zero dynamics in Theorem 3 translates in a stronger notion of minimum-phase behavior than the one of definition 4. In practice, however, the stability condition of Theorem 3 is rather difficult to check, at least analytically. On the other hand, under an appropriate choice of $\beta_{i k}$, the outputs and their derivatives not only will be exponentially decaying, but they will also get arbitrarily close to zero in finite time. Hence, for practical purposes the internal stability of the closedloop system will depend on the stability characteristics of the unforced zero dynamics. A nonlinear stability analysis can then be used to determine the region in state-space that corresponds to asymptotically stable unforced zero dynamics and, consequently, an internally stable closed-loop system. Local results can easily be obtained by checking the eigenvalues of the linear approximation of the unforced zero dynamics, around the equilibrium. If they all have negative real parts, then the closedloop system will be locally internally asymptotically stable. If there is an eigenvalue with positive real part, then the closedloop system will be internally unstable.

\section{Acknowledgment}

Financial support from the National Science Foundation, Grant No. CTS-8912836, is gratefully acknowledged.

\section{Notation}

$$
\begin{aligned}
& C_{A}=\text { molar concentration of species } A / \text { monomer } \\
& \text { in reactor } \\
& C_{A 0}=\text { molar concentration of species } A \text { in inlet } \\
& \text { stream } \\
& C_{A i n}=\text { molar concentration of monomer in mon- } \\
& \text { omer inlet stream } \\
& C_{B}=\text { molar concentration of species } B \text { in reactor } \\
& C_{l}=\text { molar concentration of initiator }
\end{aligned}
$$

$C_{\text {Rin }}=$ molar concentration of initiator in initiator inlet stream

$c_{p}=$ heat capacity of reacting mix ure

$D_{0}=$ molar concentration of dead polymer chains

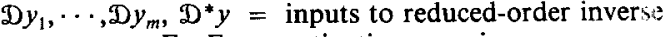

$E_{1}, E_{2}=$ activation energies

$E_{f a}, E_{P}, E_{T c}$, = activation energies for chain transfer to

$E_{T d}, E_{I}$ monomer, propagation, termination by coupling, termination by disproportionation, and initiation reactions

$f=$ vector field

$F=$ total volumetric flow rate inte reactor

$F_{I}=$ volumetric flow rate of inlet initiator stream

$g_{j}=$ vector field

$h_{i}=$ scalar field

$-\Delta H_{1},-\Delta H_{2},=$ heats of reaction

$-\Delta H_{3}$

$-\Delta H_{P}=$ heat of propagation reaction

$k_{\mathrm{t}}, k_{2}, k_{3}=$ rate constants

$k_{f a}, k_{P}, k_{T c}$, = rate constants for chain trans/er to mon-

$k_{T d}, k_{I} \quad$ omer, propagation, termination by coupling, termination by disproportionation, and initiation reactions

$P_{0}=$ molar concentration of live polymer chains

$Q=$ heat input

$r_{i}=$ relative order of output $y_{i}$ with respect to manipulated input vector

$R=$ ideal gas constant

$s$ = Laplace domain variable

$t=$ time

$T=$ temperature

$T_{i n}=$ temperature of inlet streams

$u=$ vector of manipulated inputs

$u_{1}, \cdots, u_{m}, u_{*}=$ inputs to forced zero dynamiss

$v=$ external input vector

$V=$ volume

$x=$ vector of state variables

$y_{i}=$ output variable

$Z_{1}, Z_{2}, Z_{3}=$ frequency factors in Arrhenius equation

$Z_{f a}, Z_{P}, Z_{T c}$, $=$ frequency factors in Arrhenius equation

$Z_{T d}, Z_{I}$ for chain transfer to monomer, propagation, termination by coupling, termination by disproportionation, and initiation reactions

\section{Greek letters}

$$
\begin{aligned}
\beta_{i k}= & \text { vector of parameters of input/output linear- } \\
& \text { izing control law } \\
\zeta= & \text { transformed state variables } \\
\rho & =\text { density of the reacting mixture } \\
\phi & =\text { initiator efficiency }
\end{aligned}
$$

\section{Other symbols}

$$
\begin{aligned}
\Rightarrow= & \text { implies } \\
\mathbb{R}= & \text { real line } \\
\mathbb{R}^{n}= & n \text {-dimensional Euclidean space } \\
T= & \text { transpose } \\
L_{f} h_{i}= & \text { Lie derivative of scalar field } h_{i} \text { with respect } \\
& \text { to vector field } f \\
L_{f}^{k} h_{i}= & k \text { th order Lie derivative of scalar field } h_{i} \\
& \text { with respect to vector field } f
\end{aligned}
$$

\section{Literature Cited}

Byrnes, C. I., and A. Isidori, “Global Feedback Stabilization of Nonlinear Systems," Proc. 24th IEEE CDC, Ft. Lauderdale, 1031 (1985).

Byrnes, C. I., and A. Isidori, "Local Stabilization of Minimum-phase Nonlinear Systems,"' Syst. Contr. Lett., 11, 9 (1983).

D'Andrea, B., and L. Praly, "About Finite Nonlinear Zeros for Decouplable Systems," Syst. Contr. Lett., 10, 103 (1988). 
Daoutidis, P., and C. Kravaris, "Synthesis of Feedforward/StateFeedback Controllers for Nonlinear Processes," AIChE J., 35, 1602 (1989).

Daoutidis, P., M. Soroush, and C. Kravaris, "Feedforward/Feedback Control of Multivariable Nonlinear Processes," AIChE J., 36, 1471 (1990).

Hirschorn, R. M., "Invertibility of Nonlinear Control Systems," SIAM J. Contr. Optim., 17, 289 (1979a).

Hirschorn, R. M., "Invertibility of Multivariable Nonlinear Control Systems," IEEE Trans. Auto. Contr., AC-24, 855 (1979b).

Holt, B. R., and M. Morari, "Design of Resilient Processing Plants. VI: The Effect of Right-Half-Plane Zeros on Dynamic Resilience," Chem. Eng. Sci., 40, (1) 59 (1985).

Isidori, A., Nonlinear Control Systems: An Introduction, 2d Ed. Springer, Berlin-Heidelberg (1989).

Isidori, A., and C. H. Moog, "On the Nonlinear Equivalent of the Notion of Transmission Zeros," Modeling and Adaptive Control, C. I. Byrnes, A. H. Kurzhanski, eds. Lec. Notes Contr. Info. Sci. 105, Springer (1988).

Kravaris, C., "Input/Output Linearization: A Nonlinear Analog of Placing Poles at the Process Zeros," AIChE J., 34, 1803 (1988).

Kravaris, C., and C. B. Chung, "Nonlinear State Feedback Synthesis by Global Input/Output Linearization,' AIChE J., 33, 592 (1987).

Kravaris, C., and M. Soroush, "Synthesis of Multivariable Nonlinear Controllers by Input/Output Linearization," AIChE J., 36, 249 (1990).

MacFarlane, A. G. J., and N. Karcanias, "Poles and Zeros of Linear Multivariable Systems: A Survey of the Algebraic, Geometric and Complex Variable Theory,"' Int. J. Contr., 24, 33 (1976).

Van de Vusse, J. G., "Plug-Flow-Type Reactor versus Tank Reactor," Chem. Eng. Sci., 19, 994 (1964).

\section{Appendix: Proofs of Theorems Proof of Theorem 1}

A constructive proof of Theorem 1 through Hirschorn's inversion algorithm is possible, but is not given here because of the rather complicated procedure and the technicalities involved. Instead, we prove Theorem 1 by simply verifying that the system given by Eq. 5 indeed acts as an inverse to the original system.

In particular, given the outputs $y_{i}$ for the system described by Eq. 1, and calculating expressions for their derivatives until the control vector appears explicitly, we get:

$$
\begin{aligned}
\frac{d^{r_{1}} y_{1}}{d t^{r_{1}}} & =L_{f}^{r_{1}} h_{1}(x)+L_{g} L_{f}^{r_{1}-1} h_{1}(x) u \\
& \vdots \\
\frac{d^{r_{m}} y_{m}}{d t^{r_{m}}} & =L_{f}^{r_{m}} h_{m}(x)+L_{g} L^{r_{m}-1} h_{m}(x) u
\end{aligned}
$$

Since the characteristic matrix is nonsingular, the above set of equations can be solved for $u$ to obtain:

$$
u=C(x)^{-1}\left(\left[\begin{array}{c}
\frac{d^{r_{1}} y_{1}}{d t^{r_{1}}} \\
\vdots \\
\frac{d^{r_{m}} y_{m}}{d t^{r_{m}}}
\end{array}\right]-\left[\begin{array}{c}
L_{f}^{r_{1}} h_{1}(x) \\
\vdots \\
L_{f}^{r_{m}} h_{m}(x)
\end{array}\right]\right)
$$

subject to the dynamics

$$
\dot{x}=f(x)+g(x) C(x)^{-1}\left(\left[\begin{array}{c}
\frac{d^{r_{1}} y_{1}}{d t^{r_{1}}} \\
\vdots \\
\frac{d^{r_{m}} y_{m}}{d t^{r_{m}}}
\end{array}\right]-\left[\begin{array}{c}
L_{f}^{r_{1}} h_{1}(x) \\
\vdots \\
L_{f}^{r_{m}} h_{m}(x)
\end{array}\right]\right)
$$

But the $u$ calculated above is exactly equal to the output of the dynamic system described by Eq. 5 (just substitute $x$ for $\xi)$. Therefore, by definition of the inverse, Eq. 5 is a realization of the inverse of the original system.

\section{Proof of Theorem 2}

Applying the inversion formula given by Eq. 5 for the system in the normal form of Eq. 7, we obtain the following inverse system:

$$
\dot{\zeta}_{1}^{(0)}=F_{1}(\zeta)+G_{1}(\zeta) C(\zeta)^{-1}\left(\left[\begin{array}{c}
\frac{d^{r_{1}} y_{1}}{d t^{r_{1}}} \\
\vdots \\
\frac{d^{r_{m} y_{m}}}{d t^{r_{m}}}
\end{array}\right]-\left[\begin{array}{c}
W_{1}(\zeta) \\
\vdots \\
W_{m}(\zeta)
\end{array}\right]\right)
$$

$$
\dot{\zeta}_{n-\Sigma_{i} r_{1}}^{(0)}=F_{n-\Sigma_{r_{1}}}(\zeta)+G_{n-\Sigma_{i} r_{i}}(\zeta) C(\zeta)^{-1}\left(\left[\begin{array}{c}
\frac{d^{r_{1}} y_{1}}{d t^{r_{1}}} \\
\vdots \\
\frac{d^{r_{m}} y_{m}}{d t^{r_{m}}}
\end{array}\right]-\left[\begin{array}{c}
W_{1}(\zeta) \\
\vdots \\
W_{m}(\zeta)
\end{array}\right]\right)
$$

$$
\begin{gathered}
\dot{\zeta}_{1}^{(1)}=\zeta_{2}^{(1)}=\frac{d y_{1}}{d t} \\
\vdots \\
\dot{\zeta}_{r_{1}-1}^{(1)}=\zeta_{r_{1}}^{(1)}=\frac{d^{r_{1}-1} y_{1}}{d t^{r_{1}-1}} \\
\dot{\zeta}_{r_{1}}^{(1)}=\frac{d^{r_{1}} y_{1}}{d t^{r_{1}}}
\end{gathered}
$$

$$
\begin{gathered}
\dot{\zeta}_{1}^{(m)}=\zeta_{2}^{(m)}=\frac{d y_{m}}{d t} \\
\vdots \\
\dot{\zeta}_{r_{m}-1}^{(m)}=\zeta_{r_{m}}^{(m)}=\frac{d^{r_{m}-1} y_{m}}{d t^{r_{m}-1}} \\
\dot{\zeta}_{r_{m}}^{(m)}=\frac{d^{r_{m}} y_{m}}{d t^{r_{m}}}
\end{gathered}
$$




$$
u=C(\zeta)^{-1}\left(\left[\begin{array}{c}
\frac{d^{r_{1}} y_{1}}{d t^{r_{1}}} \\
\vdots \\
\frac{d^{r_{m} y_{m}}}{d t^{r_{m}}}
\end{array}\right]-\left[\begin{array}{c}
W_{1}(\zeta) \\
\vdots \\
W_{m}(\zeta)
\end{array}\right]\right)
$$

By viewing the outputs $y_{i}$ and their derivatives as inputs to the first $\left(n-\Sigma_{i} r_{i}\right)$ equations and using the notation introduced in Eq. 9, we obtain exactly the realization given by Eq. 10 .

Manuscript received Dec. 19, 1989, and revision received Jan. 17, 1991. 